\title{
¿Y esto qué es? ¿Por qué...? El desarrollo de unidades de discurso explicativas durante la lectura de cuentos en el hogar
}

What is this? Why...? The development of explanatory units of speech during storybook reading at home

\author{
Alejandra Stein* \\ Ailín Paula Franco Accinelli** \\ Eliana Belén González Lynn***
}

\section{Resumen}

Se propone contribuir al conocimiento de las características del habla explicativa producida en 39 situaciones de lectura de cuentos en hogares de 13 niños (30-42 meses) de sectores medios de Argentina. Las unidades explicativas en los intercambios en torno al cuento fueron analizadas según el tipo de explicación, quién las inicia y produce, y su nivel de distanciamiento respecto del entorno inmediato de la situación. Los resultados evidencian el potencial de las lecturas de cuentos para la producción de habla explicativa compleja de manera compartida entre el niño y el adulto. Se identificaron diferencias longitudinales y según la familiaridad con el cuento leído. Se destacan las implicancias pedagógicas para el diseño de intervenciones educativas
\end{abstract}

orientadas a promover el dominio de discurso extendido.

Palabras clave: desarrollo discursivo, lectura de cuentos, explicación, infancia

Abstract
The aim of the study is to contribute to
the knowledge of the characteristics of the
explanatory speech produced during 39
storybook reading in 13 middle income
argentinean homes when children were
30 and 42 months old. The explanatory
discourse units produced during the
exchanges about the story were analyzed
according to the type of explanation, who
initiated and produced them, and their level
of distance from the immediate environment
of the situation. Results showed the potential
of storybook reading for the production of

* Consejo Nacional de Investigaciones Científicas y Técnicas de Argentina. Centro Interdisciplinario de Investigaciones en Psicología Matemática y Experimental ; Universidad de Buenos Aires. Facultad de Filosofía y Letras. Mail de contacto: alejandrastein@yahoo.com.ar ** Consejo Nacional de Investigaciones Científicas y Técnicas de Argentina. Centro Interdisciplinario de Investigaciones en Psicología Matemática y Experimental.

*** Universidad de Buenos Aires. Facultad de Filosofía y Letras.

DOI: https://doi.org/10.46553/RPSI.17.33.2021.p81-103

Fecha de recepción: 21 de febrero de 2021 - Fecha de Aceptación: 11 de mayo de 2021 
complex explanatory speech in comparison with play and meal situations. Differences were found according to children's age and to familiarity with the story read. The pedagogical implications of the study are highlighted.

Keywords: discursive development, storybook reading, explanation, infancy

\section{Introducción}

El presente estudio se propone contribuir al conocimiento del desarrollo discursivo, en particular, la adquisición de la explicación como forma de discurso extendido durante la lectura de cuentos en el hogar en el período comprendido entre los 2.6 y los 3.6 años. El dominio de diferentes formas de discurso extendido constituye un aspecto fundamental del desarrollo lingüístico y cognitivo infantil (Griffin et al., 2004). Asimismo, está estrechamente relacionado con el proceso de alfabetización (Snow \& Beals, 2006), la adquisición del lenguaje académico (Snow, 2014) y la escolarización (Heller, 2014).

Diversos autores en el campo de la psicología y la psicolingüística han definido el discurso extendido como una forma de discurso que se extiende de manera coherente en torno a un mismo tópico a lo largo de varios turnos (Blum-Kulka, 2002; Pan \& Snow, 1999; Snow \& Uccelli, 2009).

Las conversaciones "cara a cara" son la primera forma de discurso extendido en la que los niños se involucran (Pan \& Snow, 1999). Progresivamente, van construyendo conocimientos y habilidades que les permiten comprender y producir variadas formas de discurso extendido, tales como producir narraciones, contar chistes, elaborar argumentos y proporcionar explicaciones. Para ello, los niños deben dominar una serie de habilidades lingüísticas y cognitivas tales como: considerar la perspectiva del interlocutor, expresar su propia perspectiva, identificar y asumir diferentes posicionamientos acerca de la información objeto de comunicación y conocer las convenciones relativas a las distintas unidades de discurso.

La mayoría de las investigaciones previas que estudiaron la adquisición de discurso extendido se centraron en la narración (Carmiol \& Sparks; 2014; McCabe et al., 2008; Nelson, 1989; entre muchos otros) debido a su estrecha relación con diferentes aspectos del desarrollo infantil (McCabe et al., 2008). Son menos numerosas las investigaciones que abordaron el desarrollo del discurso explicativo, a pesar de su presencia en los intercambios cotidianos durante la infancia (Aukrust, 2004; Blum-Kulka et al., 2010; Veneziano \& Sinclair, 1985) y de su relevancia para la escolarización y para el desarrollo lingüístico y cognitivo (Griffin et al., 2004; Heller, 2014; Pappas, 1993; Sepúlveda \& Álvarez-Otero, 2010; Snow, 2014; Snow \& Beals, 2006).

\section{Las situaciones de lectura de cuentos como una oportunidad para el desarrollo discursivo}

Las situaciones de lectura de cuento constituyen una oportunidad para el aprendizaje de habilidades de discurso extendido relacionadas con la narración. El texto escrito y las ilustraciones de los cuentos proporcionan una referencia compartida que favorece atención conjunta (Ninio \& Bruner, 1978; Keleman et al., 2014) y una base común para la construcción compartida de un discurso que se extiende en torno a un 
¿Y esto qué es? ¿Por qué...? El desarrollo de unidades de discurso explicativas...

mismo tema por varios turnos.

Una importante línea de investigación se centró en el estudio de la interacción durante las situaciones de lectura en el contexto de la familia a través de los cuales tiene lugar la construcción compartida de discurso extendido.

Las investigaciones realizadas en esta línea mostraron que, con frecuencia, las situaciones de lectura de cuentos en el hogar se caracterizan por ser altamente dialogadas. Los adultos - mayormente la madre en los estudios realizados - no se limitan a realizar una lectura literal del texto, sino que introducen diversas modificaciones a través de las cuales crean un contexto cognitivo que favorece la comprensión (Haden et al., 1996; Ninio \& Bruner, 1978; Stein \& Rosemberg, 2012).

En su investigación con familias anglo y afroamericanas de Estados Unidos, Heath (1983) observó que, durante la lectura, las madres formulaban preguntas que daban lugar a explicaciones que responden a “¿qué?" (what explanations). Estos interrogantes estaban orientados a facilitar por parte de los niños el ordenamiento de los eventos del cuento, la identificación de las ideas principales, entre otros aspectos. Las madres realizaban también intervenciones relacionadas con las motivaciones de los personajes o las razones de la ocurrencia de un evento (reason explanations). La demanda de predicciones identificada por Wells (1985) constituye una variación de este tipo de explicación, en tanto el niño debe comprender las razones que subyacen a un evento para poder anticipar eventos futuros.

Algunos trabajos mostraron que las intervenciones de los adultos en torno al cuento se modifican con la edad de los niños.
Estos estudios - mayormente realizados con familias caucásicas de sectores mediosmostraron que progresivamente las madres incrementan el nivel de dificultad de sus intervenciones (Ninio \& Bruner, 1978; Van Kleeck et al., 1997). Inicialmente, lexicalizan objetos y acciones $\mathrm{y}$, posteriormente, requieren que los niños anticipen lo que sucederá en la historia. Otros trabajos con población afroamericana también mostraron variaciones en los estilos de lectura maternos según la edad: con los más pequeños, las madres nombraban y señalaban los objetos ilustrados, en tanto que con los mayores esperaban que escucharan el cuento en silencio (Heath, 1983; Heath \& Thomas, 1984).

Sulzby y Teale (1987) analizaron, en particular, las variaciones longitudinales durante la lectura repetida de un mismo cuento. En estos casos, no observaron que los niños se mantuvieran en silencio con la edad; por el contrario, se registró un mayor protagonismo infantil en la narración asociado a un cambio en los roles durante la lectura. Con el incremento de la edad, durante la lectura de un cuento conocido, los niños nombraban las ilustraciones y formulaban preguntas. Además, los participantes se involucraban en intercambios acerca de las características de los objetos y personajes, introducían sinónimos de las palabras presentes en el texto y establecían relaciones entre la historia y experiencias personales. En esta misma línea, Haden et al. (1996) analizaron longitudinalmente la interacción en situaciones de lectura de cuentos en las que participaron díadas madre-niño de sectores medios hablantes de inglés de Estados Unidos cuando los niños tenían 40 y 58 meses de edad. El análisis 
¿Y esto qué es? ¿Por qué...? El desarrollo de unidades de discurso explicativas...

se centró en los comentarios extratextuales durante la lectura de un cuento familiar y no familiar en los dos momentos considerados. Los resultados permitieron identificar diferentes estilos de lectura maternos que se mantuvieron relativamente consistentes a lo largo del tiempo - aunque en todos los casos disminuyó el etiquetamiento con la edad-, pero no según el libro leído fuera familiar o no familiar para los niños. Otros trabajos que también analizaron la interacción durante la lectura de cuentos familiares mostraron que estas situaciones son más interactivas y que en ellas las madres demandan una mayor participación infantil (Phillips \& McNaughton, 1990; Goodsitt et al., 1988).

Como se desprende de las investigaciones mencionadas, las intervenciones de los adultos en torno a la lectura son diversas y pueden diferir en cuanto a su complejidad y grado de distanciamiento respecto de las ilustraciones y el texto del cuento. Mientras algunas intervenciones se centran en el etiquetamiento y la descripción de personajes y objetos presentes en el libro leído, otras pueden contribuir al uso de habla no inmediata relacionada con entidades y eventos no presentes en el contexto físico de la situación. Este último tipo de intervenciones resulta de especial interés debido a su contribución para el desarrollo cognitivo (Peralta \& Salsa, 2001), así como para el desarrollo del lenguaje y de la alfabetización (Beals, 1993).

Siguiendo la propuesta de Sigel (2002), algunos trabajos analizaron las intervenciones maternas durante la lectura de acuerdo con su nivel de distanciamiento respecto del entorno inmediato. Desde esta perspectiva, las estrategias de nivel alto incluyen la elaboración de conclusiones, la inferencia de relaciones causa-efecto, la anticipación y evaluación de consecuencias, la inferencia de emociones y estados internos, entre otras. Las estrategias de nivel bajo comprenden el etiquetamiento y la descripción de objetos, acciones y personajes. Las estrategias de nivel medio exigen al niño una elaboración más compleja sobre el contexto inmediato y comprenden, por ejemplo, el establecimiento de relaciones entre el texto y las ilustraciones con experiencias de la vida cotidiana, el despliegue de una situación dramática o de simulación, o el establecimiento de relaciones entre objetos.

Peralta y Salsa (2001) analizaron la interacción verbal materno-infantil en torno a un libro con imágenes y sin texto en 22 díadas madre-niño de 20 a 24 meses de edad de nivel socioeconómico bajo y medio de Argentina. Ambos grupos de madres privilegiaban estrategias de nivel bajo. Sin embargo, las madres de sectores medios presentaron un estilo más elaborado, demandante e indirecto; los niños de este grupo, por su parte, se mostraron más activos verbalmente.

En síntesis, las investigaciones revisadas mostraron que las lecturas de cuentos que favorecen el desarrollo discursivo se caracterizan por ser dialogadas. Los adultos realizan diversas intervenciones que dan lugar a distintos tipos de explicaciones con diferente grado de distanciamiento respecto del entorno inmediato. Las intervenciones adultas durante la lectura y el habla explicativa que promueven varían según la edad del niño y la familiaridad con el texto leído. Dada la relevancia de la calidad de la interacción y la complejidad del habla durante la lectura para el desarrollo, 
cabe preguntarse por las características de las explicaciones en torno al cuento, su nivel de distanciamiento, así como el papel del niño y del adulto en su producción en una población poco estudiada como es la población hispanohablante de nuestro país. Asimismo, resulta de interés analizar eventuales variaciones longitudinales en los aspectos mencionados según la familiaridad del cuento leído.

\section{El desarrollo de la explicación durante la infancia: una forma de discurso extendido menos estudiada}

El habla explicativa constituye otra forma de discurso extendido relevante para el desarrollo infantil. A pesar de ello, ha sido menos estudiada que la narración.

De manera general, las unidades de discurso explicativas durante la interacción se caracterizan por la presencia de un participante que indica que no ha comprendido algo o en los que el hablante asume que sabe algo que el destinatario necesita saber. El hablante ofrece un segmento en el que da cuenta del fenómeno en cuestión -información sobre causas y efectos, el qué y el cómo de objetos y eventos (Barbieri et al., 1990; Blum-Kulka et al., 2010). Las explicaciones permiten establecer y/o explicitar relaciones entre objetos, intenciones, eventos $\mathrm{y} / \mathrm{o}$ conceptos que pueden estar presentes o no en el contexto físico inmediato (Beals, 1993).

La participación de los niños en situaciones donde producen junto con otros esta forma de discurso promueve procesos de co-construcción dialógica de conocimientos (Blum-Kulka, 2002) y conlleva un impacto positivo en el desarrollo lingüístico y cognitivo, la alfabetización, el aprendizaje del lenguaje académico y la escolarización (Pappas, 1993; Peets \& Bialystock, 2015; Ricard \& Snow, 1990; Sepúlveda \& ÁlvarezOtero, 2010).

Diversas investigaciones mostraron que los primeros usos de esta forma de discurso tienen lugar a través de las interacciones en actividades cotidianas de juego (Aukrust, 2004), lectura de cuentos y textos expositivos, así como en conversaciones durante situaciones de comida (Aukrust \& Snow, 1998; Beals, 1993; Blum-Kulka, 2002; Stein et al., 2020).

Investigaciones con niños hablantes de inglés y francés han mostrado que las primeras explicaciones se producen antes de los 2 años. Inicialmente, los niños producen explicaciones y justificaciones de su propio comportamiento, en el marco de actos de pedido y rechazo (Veneziano \& Sinclair, 1985). Alrededor de los 3 años, los niños demandan y producen explicaciones no solo con el fin de controlar o actuar sobre los fenómenos de su vida cotidiana, sino también para conocer acerca del mundo (Callanan \& Oakes, 1992; Crowley et al., 2001; Hickling \& Wellman, 2001; Legare \& Clegg, 2014). Las explicaciones infantiles tempranas se referían primordialmente a estados y actividades humanas, pero también a animales y objetos físicos (Hickling \& Wellman, 2001).

Una serie de estudios se focalizaron específicamente en el análisis de las explicaciones durante las situaciones de comida (Aukrust \& Snow, 1998; Beals, 1993; Beals \& Snow, 1994; Blum-Kulka, 2002) con niños de 3 a 5 años. Entre los principales resultados de estas investigaciones, se observó que el habla explicativa representaba el $15 \%$ de las emisiones durante la 
conversación en familias norteamericanas de nivel socioeconómico bajo (Beals, 1993). Asimismo, se identificaron diferentes tipos de habla explicativa según su contenido y la pregunta que permitía responder. Se observó que el 49,5\% de las explicaciones se referían a las intenciones que fundamentaban acciones y directivas, seguidas de las explicaciones causales $(17,5 \%)$, referidas a estados internos $(13,3 \%)$, definiciones I descripciones (6,7\%), evidenciales $(5,4 \%)$, intenciones que subyacen a una pregunta $(4 \%)$, explicaciones referidas a procedimientos $(2 \%)$, y explicaciones que expresan relaciones de consecuencia $(1,5 \%)$ (Beals, 1993). Las explicaciones abarcaban diversos dominios - convenciones sociales, objetos físicos, estados internos y comportamientos, política, entre otrosy se ubicaban en un continuum entre explicaciones relativas al aquí y el ahora de la conversación y otras más alejadas temporal y espacialmente (Blum-Kulka, 2002). Por otra parte, se observó que eran mayormente los adultos quienes asumían el rol de explicar. En la iniciación de las secuencias, los adultos y los niños se desempeñaban de modo similar (Blum-Kulka, 2002). En estas investigaciones no se llevó a cabo un análisis longitudinal del habla explicativa ni de la participación infantil en su producción.

Aukrust (2004) analizó el habla explicativa producida por niños de 5 años durante situaciones de juego entre pares en jardines de infantes noruegos. Los niños producían diversos tipos de explicaciones, tanto de manera espontánea como en respuesta al pedido de otro niño. Los resultados mostraron que el $47,6 \%$ de los enunciados explicativos estaban anclados en la actividad inmediata.
A pesar de que la narración y la explicación se entrelazan en las interacciones cotidianas, la mayoría de las investigaciones ha abordado su estudio de manera aislada. Son escasos los trabajos que consideraron las relaciones entre ambas formas de discurso y su emergencia conjunta los intercambios cotidianos. En esta línea, el estudio de Beals y Snow (1994) centrado en las interacciones durante situaciones de comida en hogares de niños estadounidenses de 3 a 5 años, mostraron que las unidades de discurso narrativas y explicativas aparecen imbricadas en la conversación. A los 4 años predominan las unidades explicativas, aunque su extensión es menor que las narrativas. Se observó que mientras las emisiones de la madre eran más extensas en las explicaciones, los niños producían turnos más largos en las narraciones. No se observaron diferencias significativas según la edad de los niños. En un estudio posterior, Aukrust y Snow (1998) analizaron la producción de narraciones y explicaciones por parte de niños y padres de familias noruegas y estadounidenses durante las comidas. En los hogares noruegos se producían con mayor frecuencia unidades narrativas en torno a temáticas relativas a desviaciones de los guiones sociales. En las familias estadounidenses se produjeron más explicaciones relacionadas con eventos físicos o comportamientos individuales. En Argentina, Stein et al. (2020) analizaron la estructura y el anclaje interaccional de las narraciones, explicaciones y argumentaciones producidas durante situaciones de comida en los hogares de 11 niños de cuatro años de sectores medios. Se observó que, si bien las tres formas de discurso se entrelazaban en las conversaciones, las narraciones eran más frecuentes y extensas que las 
¿Y esto qué es? ¿Por qué...? El desarrollo de unidades de discurso explicativas...

explicaciones. La narración era mayormente desencadenada a partir del tópico de la conversación, mientras que la explicación y la argumentación se desencadenaban a partir de objetos o acciones presentes en la situación. Los niños desempeñaban un rol activo en la producción de narraciones, mientras que en mayor medida escuchaban la producción de explicaciones por parte de sus interlocutores.

Desde una perspectiva cognitiva, en el marco de la línea de investigación en torno a la enseñanza de la ciencia, se han llevado a cabo en los últimos años estudios con intervención en los que se buscó promover el habla explicativa y la comprensión de explicaciones científicas a través de la lectura de textos narrativos (Kelemen et al, 2014; Leech et al., 2020; Legare et al., 2013). De manera general, estos estudios, llevados a cabo con niños a partir de 4 años, mostraron un impacto positivo de la inclusión de explicaciones en textos narrativos, así como de la cantidad de habla explicativa en los intercambios en torno a la lectura en el aprendizaje de conocimientos científicos.

\section{El presente estudio}

A partir de considerar las investigaciones antecedentes, el presente estudio se propone contribuir al conocimiento de las características que presenta el habla explicativa durante la lectura de cuentos en hogares de niños de sectores medios de Argentina durante el período comprendido entre los 30 y los 42 meses de edad. Específicamente, se busca responder a las siguientes preguntas:

¿Qué cantidad de habla explicativa producen los participantes en los intercambios en torno a la lectura? ¿Cuál es el papel del niño y del adulto en su producción?

¿Qué tipo de unidades de discurso explicativas se producen?

¿Cuál es el nivel de distanciamiento de las unidades de discurso explicativas?

¿Qué variaciones longitudinales se producen a los 42 meses en los aspectos mencionados (cantidad de habla explicativa, papel del niño y del adulto, tipo y nivel de distanciamiento de las explicaciones) durante la lectura del mismo cuento que a los 30 meses? ¿Qué variaciones longitudinales se producen durante la lectura de un cuento nuevo (no familiar)?

\section{Metodología}

\section{Corpus y participantes}

Se analizaron 39 situaciones de lectura de cuento registradas mediante audio en los hogares de 13 niños (cinco niñas) de sectores medios de la Ciudad de Buenos Aires, Argentina cuando tenían 2.6 años (m: 30,3 meses; sd: 0,48) y 3.6 años (m: 42,2 meses; sd: 0,43). La totalidad de los niños y sus familiares son monolingües hablantes de español. En las situaciones de lectura participaron el niño y su madre; en dos situaciones estuvo también presente un hermano mayor. Los niños asistían al jardín de infantes en el momento de realización del estudio. Cada situación de lectura tuvo una duración aproximada de 5:15 minutos (rango: 1-12).

\section{Procedimientos de obtención de la información empírica}

Se tuvo acceso a las familias a través de la red personal de los investigadores. Los adultos firmaron consentimientos informados por 
ellos y por sus hijos en los que se estableció un acuerdo de anonimato y confidencialidad. Los niños tenían conocimiento acerca de la grabación de las situaciones de lectura. La investigación cumple con los lineamientos para el comportamiento ético establecidos por el Consejo Nacional de Investigaciones Científicas de Argentina (CONICET, 2006).

La primera autora del trabajo realizó una visita al hogar de cada niño cuando estos tenían 2.6 y 3.6 años1. En cada visita se indujeron las situaciones de lectura, realizadas conjuntamente por el niño y un miembro del hogar. Para facilitar la comparabilidad de la información empírica, se controló el material de lectura - el cuento ilustrado. Para la selección de los dos cuentos utilizados se atendió a que los contenidos fueran familiares para los niños y que la historia tuviera una estructura canónica. Los títulos usados fueron: Ardilla tiene hambre (Kitamura, 1998) y El perro y el gato se ensucian (traducción de la primera autora de Cat and dog in a mess, Rayner, 2006). Durante la visita a los 2.6 años se llevó a cabo la lectura del cuento Ardilla tiene hambre (13 situaciones); en la segunda visita (3.6 años) se repitió la lectura del mismo cuento y se registró además la lectura de un cuento nuevo El perro y el gato se ensucian (26 situaciones). Con el objeto de resguardar la validez ecológica de los datos, no se pautó al interlocutor del niño, la extensión de la situación ni cómo interactuar con los niños.

Las transcripciones siguen las pautas del formato CHAT (Codes for the Human Analysis of Transcripts) que permite el procesamiento de los datos con el programa CLAN (Computerized Language
Analysis; MacWhinney, 2000).

\section{Identificación de las unidades de discurso explicativas}

Se identificaron las unidades explicativas producidas en el marco de los intercambios en torno al cuento - no se consideraron las eventuales explicaciones presentes en el texto original de cada libro. Para la identificación de las explicaciones, se siguió la propuesta de Quasthoff et al. (2017), quienes reconocen en la interacción unidades que constituyen bloques de construcción coherentes cuyo inicio y finalización es marcada por los propios participantes. Las unidades de discurso explicativas se producen cuando un participante indica que no ha comprendido algo, o bien el hablante asume que sabe algo que el destinatario necesita saber. El hablante da cuenta del fenómeno en cuestión, que puede consistir en una explicación causal o de finalidad, así como segmentos orientados a proporcionar información acerca del qué y el cómo de objetos y eventos (Barbieri et al., 1990; Beals, 1993; Blum-Kulka et al., 2010). Las explicaciones permiten establecer $y / o$ explicitar relaciones entre objetos, intenciones, eventos, conceptos o conclusiones.

Se identificaron 94 unidades de discurso explicativas en las 13 situaciones de lectura del cuento Ardilla tiene hambre a los 2.6 años (m: 7,23 de: 3,8); 102 unidades explicativas en las 13 situaciones de lectura de ese mismo cuento registradas a los 3.6 años (m: 7,84 de: 6,5) y 83 unidades en las 13 situaciones de lectura (m: 6,38 sd: 4,2) del cuento El perro y el gato se ensucian cuando los niños tenían 3.6 años. 
¿Y esto qué es? ¿Por qué...? El desarrollo de unidades de discurso explicativas...

\section{Codificación de las unidades de discurso explicativas}

Las unidades de discurso explicativas fueron codificadas según el tipo de explicación, quién las inicia y produce, y según su nivel de distanciamiento respecto del entorno inmediato de la situación.
Tipo de unidad explicativa

Se distinguieron los tipos de explicaciones presentados en la Tabla 1 (Beals, 1993).

\section{Inicio de la unidad explicativa}

Se indentificó el participante —madre o

\section{Tabla 1}

Tipos de unidades explicativas

\begin{tabular}{|c|c|c|}
\hline Tipo & Definición & Ejemplo \\
\hline Causalidad $^{2}$ & $\begin{array}{l}\text { Relaciones de causa - efecto. } \\
\text { Comprende las explicaciones } \\
\text { que responden a: ¿Por qué } \\
\text { sucedió X? ¿Cuáles son las } \\
\text { consecuencias de X? }\end{array}$ & $\begin{array}{l}\text { *MAD: la ardilla no tiene dónde } \\
\text { guardar su nuez. } \\
\text { *NIÑ: para que nadie la agarre. } \\
\text { *MAD: claro porque si alguien la } \\
\text { agarra se queda sin nuez y tiene que } \\
\text { buscar otra. }\end{array}$ \\
\hline Evidencia & $\begin{array}{l}\text { Evidencia de un determinado } \\
\text { conocimiento. Responde a la } \\
\text { pregunta: ¿Cómo se sabe X? }\end{array}$ & $\begin{array}{l}\text { *MAD: cómo te diste cuenta que la } \\
\text { guarida estaba abajo de la tierra? } \\
\text { *NIÑ: porque viven ahí. *MAD: } \\
\text { porque vos sabés que los topos viven } \\
\text { abajo de la tierra? } \\
\text { *NIÑ: sí. }\end{array}$ \\
\hline Intención & $\begin{array}{l}\text { Intención que subyace a una } \\
\text { acción. Responde a la pregunta: } \\
\text { ¿Por qué X lleva a cabo } \\
\text { determinada acción? }\end{array}$ & $\begin{array}{l}\text { *NIÑ: la ardilla la [refiere a la } \\
\text { nuez] escondió en un agujero para } \\
\text { comerla. }\end{array}$ \\
\hline Estado interno & 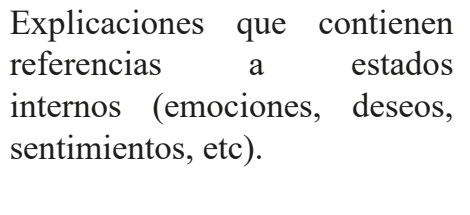 & $\begin{array}{l}\text { *MAD: y mirá (.) sale corriendo la } \\
\text { ardilla. viste? } \\
\text { *NIÑ: iba saliendo y apareció un } \\
\text { lobo. } \\
\text { *MAD: claro (.) se asustó }\end{array}$ \\
\hline Procedimiento & $\begin{array}{l}\text { Descripción } \quad \text { de } \\
\text { procedimiento. Responde } \\
\text { ¿Cómo se hace...? }\end{array}$ & $\begin{array}{l}\text { *MAD: cómo hacen los perros } \\
\text { para secarse }(.) \text { sabés? (dramatiza } \\
\text { moviendo el cuerpo) }\end{array}$ \\
\hline \multirow[t]{2}{*}{$\begin{array}{l}\text { Definición/ } \\
\text { descripción }\end{array}$} & $\begin{array}{l}\text { Definición, significado de } \\
\text { una palabra o expresión, } \\
\text { descripción de un objeto, acción } \\
\text { o personaje. Responde a: ¿Qué } \\
\text { es X? ¿Cómo es X? }\end{array}$ & $\begin{array}{l}\text { *MAD: esta es la panza (.) es la } \\
\text { barriga. } \\
\text { *MAD: qué es esto? un árbol (.) no? } \\
\text { *NIÑ: sí. }\end{array}$ \\
\hline & & $\begin{array}{l}\text { *MAD: vos no sé si comiste nuez. la } \\
\text { nuez es un frutito duro que se come }\end{array}$ \\
\hline
\end{tabular}


niño- que inició cada unidad explicativa.

\section{Elaboración de la unidad explicativa}

El desarrollo de una unidad explicativa suele ser un logro interaccional de los participantes en el intercambio (Aukrust, 2004; Quasthoff et al., 2017). En ese sentido, las unidades explicativas analizadas podían ser elaboradas por la madre, por el niño, o por ambos participantes de manera conjunta. Nivel de distanciamiento

Las unidades de discurso explicativas se codificaron según su nivel de distanciamiento respecto de información presente en el texto y/o las ilustraciones (Sigel, 2002).

El 30\% de las unidades explicativas fue codificado por la primera y la segunda autora. El porcentaje de acuerdo alcanzado para cada categoría fue del $88,3 \%$ o superior. En los casos de desacuerdo se alcanzó acuerdo luego de un chequeo.

\section{Procedimientos de análisis}

Se calculó la proporción de habla explicativa en cada una de las situaciones analizadas. Para ello, se dividió la cantidad de palabras en las unidades explicativas identificadas en cada situación de lectura sobre la

\section{Tabla 2}

Nivel de distanciamiento de las unidades explicativas

\begin{tabular}{|c|c|}
\hline Nivel & Ejemplo \\
\hline $\begin{array}{l}\text { Nivel alto. Explicaciones que implican } \\
\text { operaciones de generalización, } \\
\text { anticipaciones e inferencias. }\end{array}$ & $\begin{array}{l}\text { *NIÑ: por qué estará enojado? } \\
\text { *MAD: y porque todos dicen que los perros y } \\
\text { los gatos no son amigos (.) que se pelean. } \\
\text { *NIÑ: por qué? } \\
\text { *MAD: dicen eso pero la realidad es que hay } \\
\text { gatos y perros que sí son amigos como Frida } \\
\text { y Tita. }\end{array}$ \\
\hline $\begin{array}{l}\text { Nivel medio. Explicaciones en las que } \\
\text { se establecen relaciones entre el texto y } \\
\text { las ilustraciones con experiencias de la } \\
\text { vida cotidiana de los niños, despliegue de } \\
\text { una acción dramática, la relación con un } \\
\text { término más familiar }\end{array}$ & $\begin{array}{l}\text { *MAD: viste este perro sabés qué está } \\
\text { haciendo? te acordás cuando Lulú se moja } \\
\text { que \&shuck+shuck+shuck se sacude todo? te } \\
\text { acordás? está haciendo lo mismo. } \\
\text { *MAD: esta es la panza (.) es la barriga } \\
\text { (señala la ilustración). }\end{array}$ \\
\hline $\begin{array}{l}\text { Nivel bajo. Explicaciones centradas en } \\
\text { la descripción y denominación de los } \\
\text { objetos y eventos presentes en el texto y } \\
\text { las ilustraciones. }\end{array}$ & $\begin{array}{l}\text { *MAD: qué es esto? un árbol? *NIÑ: sí. } \\
\text { *MAD: <+[=! lee] ese hueco en el árbol es un } \\
\text { lugar perfecto>. este es el hueco (señala). } \\
\text { *NIÑ: sí (.) pero es muy chiquitito. }\end{array}$ \\
\hline
\end{tabular}


cantidad total de palabras producidas por los participantes en la situación. Para el recuento de palabras se empleó el comando freq del programa CLAN (MacWhinney, 2000).

El análisis buscó identificar diferencias longitudinales en los diversos aspectos considerados relativos al habla explicativa producida en las situaciones de lectura: proporción de habla explicativa, quién inicia y produce las unidades explicativas, tipo y nivel de distanciamiento de la unidad explicativa. Se emplearon proporciones de cada medida, tomando como base el total de unidades de discurso explicativas producidas en cada situación. En primer lugar, se llevó a cabo un análisis de las variaciones longitudinales en el habla explicativa durante la lectura de un cuento conocido (cuento Ardilla tiene hambre a los 2.6 y 3.6 años). Luego, se analizó longitudinalmente el habla explicativa durante la lectura de un cuento no familiar (cuento Ardilla tiene hambre a los 2.6 y cuento El perro y el gato se ensucian a los 3.6). Se realizó un análisis descriptivo de los datos y luego se empleó la prueba estadística no paramétrica de los rangos con signo de Wilcoxon con el fin de analizar el habla explicativa producida en los dos momentos considerados. El análisis estadístico fue realizado en R ( $\mathrm{R}$ Core Team, 2017).

\section{Resultados}

\section{Variaciones longitudinales en el habla explicativa durante la lectura de un cuento conocido}

Se observó una proporción similar de habla explicativa en los dos momentos considerados. Mientras en las situaciones de lectura registradas a los 30 meses las unidades de discurso explicativas configuran el $38 \%$ del habla producida por los participantes, en las situaciones desarrolladas a los 42 meses el habla explicativa representó un $39 \%$ del habla total.

Con respecto al papel de los participantes en los intercambios en la iniciación de las explicaciones (ver Figura 1), los resultados mostraron que tanto a los

\section{Figura 1}

Proporción de unidades explicativas iniciadas por el niño y la madre según edad
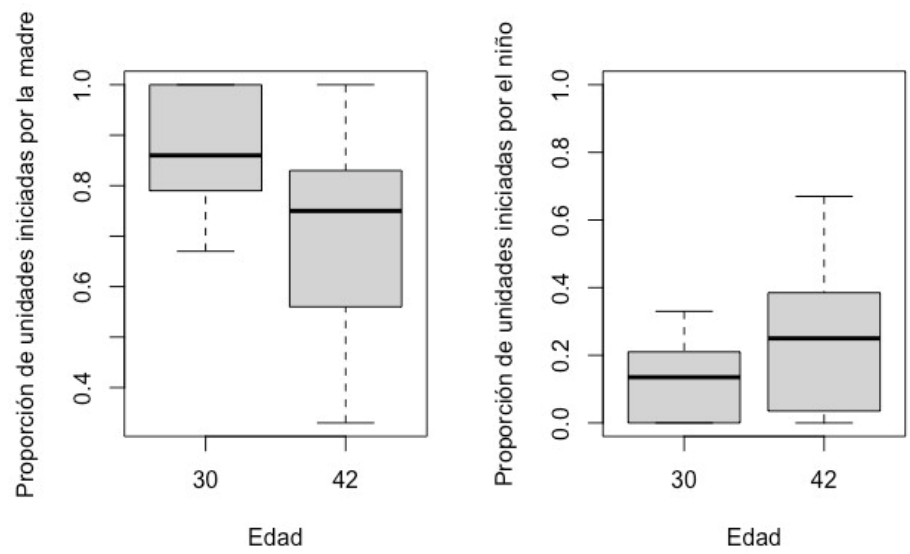

Revista de Psicología. Año 2021. Vol. 17, № 33, pp. 81-103 
30 meses como a los 42 meses las madres inician la mayor parte de las unidades de discurso explicativas. Sin embargo, el test de Wilcoxon puso de manifiesto un descenso significativo en la proporción de unidades explicativas iniciadas por la madre cuando los niños son más grandes $(\mathrm{V}=78, \mathrm{p}=.02)$. Por su parte, en los dos momentos considerados los niños inician el $30 \%$ o menos de las explicaciones. Si bien se observa un incremento en las unidades iniciadas por los niños a los 42 meses de edad, no resulta estadísticamente significativo ( $\mathrm{V}=27, \mathrm{p}=.21$ ).

El análisis también contempló el papel de los participantes en la producción de las unidades explicativas (Figura 2).

Como se observa en la Figura 2, cuando los niños tienen 42 meses se produce un descenso significativo de las unidades explicativas producidas por la madre $(\mathrm{V}=76$, $\mathrm{p}=.03$ ). Si bien se observa un incremento de las explicaciones elaboradas por los niños, así como en la proporción de unidades explicativas producidas de manera conjunta por la madre y el niño en los dos momentos, las diferencias no son estadísticamente significativas $(\mathrm{V}=21, \mathrm{p}=.14 ; \mathrm{V}=39, \mathrm{p}=.68$ respectivamente).

El análisis del tipo de explicación puso de manifiesto que las unidades explicativas más frecuentes consisten en definiciones y descripciones de los personajes, objetos y acciones presentes en el texto y en las ilustraciones (M: 0,56 DE: 0,36), seguidas de las explicaciones que expresan relaciones de causalidad (M:

\section{Figura 2}

Producción de las unidades explicativas según edad
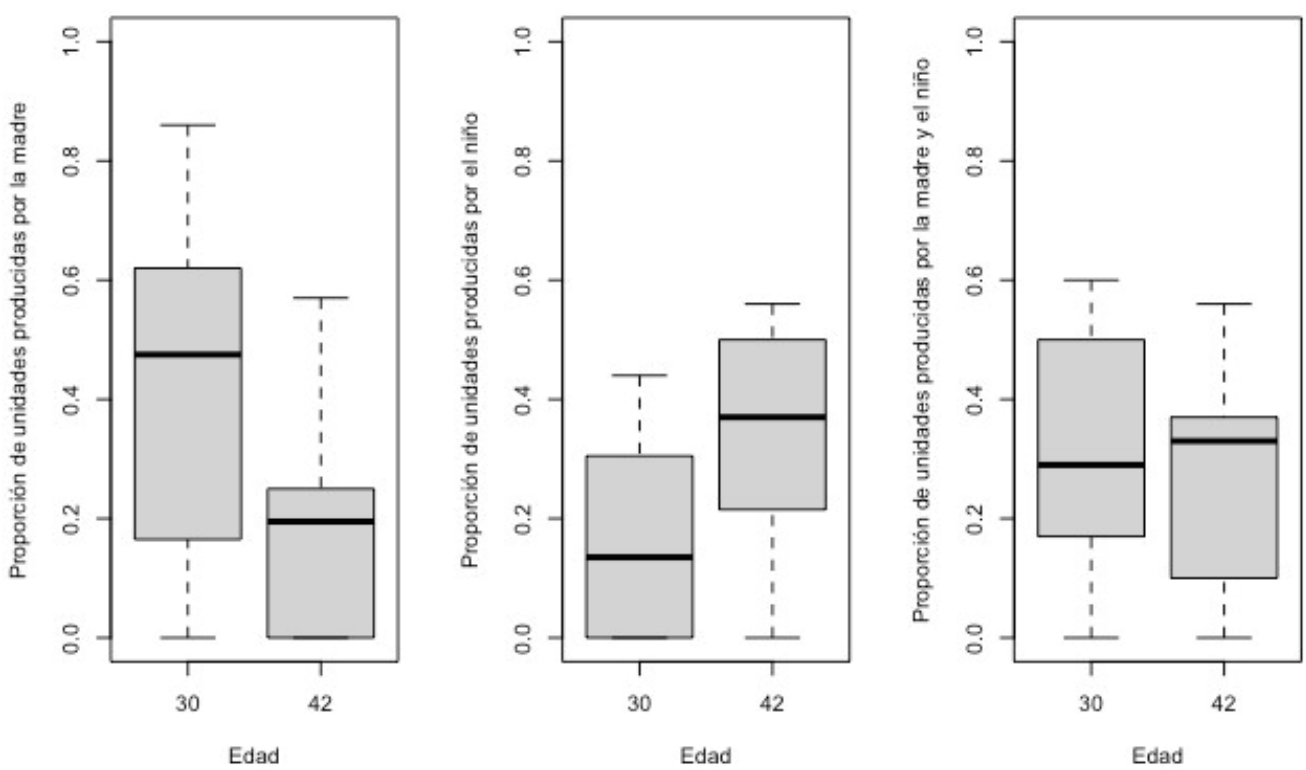

Revista de Psicología. Año 2021. Vol. 17, No 33, pp. 81-103 
¿Y esto qué es? ¿Por qué...? El desarrollo de unidades de discurso explicativas...

0,34 DE: 0,24). En conjunto, el resto de las explicaciones alcanzan una proporción de 0,1 cuando los niños tienen 30 meses de edad. A los 42 meses se produce un descenso significativo de las explicaciones del tipo definición/descripción $(\mathrm{V}=77$, $\mathrm{p}=.02$ ) cuya proporción se reduce a 0,36 en promedio (DE: 0,26). Si bien tiene lugar un incremento de las unidades explicativas que expresan relaciones de causalidad (M: 0,4 DE: 0,27), las diferencias entre los dos momentos no resultan estadísticamente significativas $(\mathrm{V}=28, \mathrm{p}=.24)$. Se mantiene una baja proporción de las explicaciones del tipo evidencial, estado interno, intención y procedimiento.

Las unidades de discurso explicativas producidas durante los intercambios en torno al cuento fueron analizadas según el nivel de distanciamiento respecto del entorno inmediato (Figura 3). La mayoría de las explicaciones producidas cuando los niños tienen 30 meses implican un bajo nivel de distanciamiento respecto del texto y las ilustraciones del cuento, seguidas de las explicaciones de nivel medio y alto en proporciones similares. Cuando los niños tienen 3.6 años, se produce un incremento de las explicaciones de nivel alto. Si bien el incremento no resulta significativo $(V=33$, $\mathrm{p}=.41$ ), este tipo de explicaciones son las más frecuentes en este segundo momento. Se identificó también un aumento de la proporción de unidades discursivas de nivel medio ( $\mathrm{V}=21, \mathrm{p}=.09)$ acompañado de un descenso significativo de las explicaciones

\section{Figura 3}

Nivel de distanciamiento de las unidades explicativas según edad
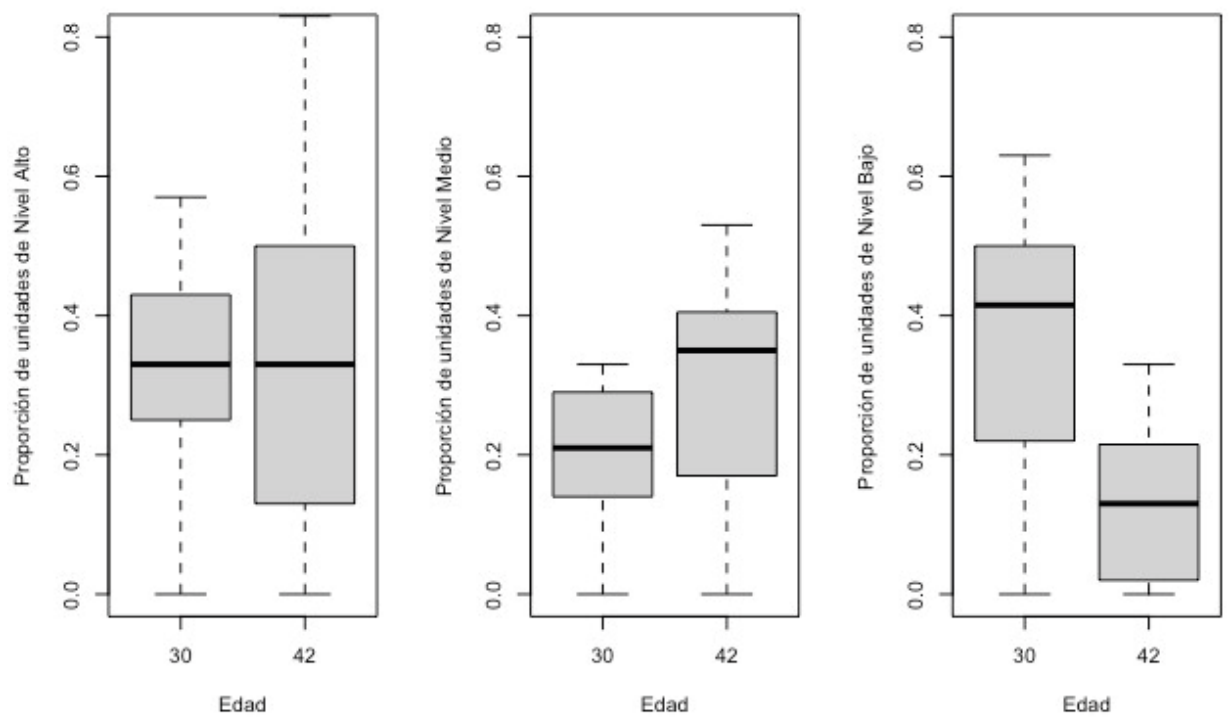

Revista de Psicología. Año 2021. Vol. 17, No 33, pp. 81-103 
de nivel bajo a los 42 meses $(\mathrm{V}=77, \mathrm{p}=.02)$.

Variaciones longitudinales en el habla explicativa durante la lectura de un cuento no familiar

No se identificaron diferencias longitudinales en la proporción de habla explicativa: cuando los niños tienen 30 meses, la proporción de este tipo de habla es en promedio 0.38 (DE: 0.22 ), mientras que a los 42 meses es de 0.34 (DE: 0.19).

Respecto del papel de los participantes en la iniciación de las unidades de discurso explicativas (Figura 4), se observó que, tanto a los 30 meses como a los 42 meses, las madres inician la mayoría de las explicaciones durante la lectura de cuentos que no son conocidos por los niños. Las diferencias entre ambos momentos no son estadísticamente significativas $(\mathrm{V}=55$, $\mathrm{p}=.54$ ). Tampoco se observaron diferencias en las unidades discursivas iniciadas por los niños entre los 30 y los 42 meses de edad $(\mathrm{V}=36, \mathrm{p}=.54)$.

La Figura 5 presenta los resultados del análisis del papel de los participantes en la producción de las explicaciones en torno a la lectura.

Como se observa en la Figura 5, durante la lectura de un cuento no conocido cuando los niños tienen 42 meses disminuye la proporción de explicaciones producidas por la madre, así como aquellas producidas por la madre y el niño de manera conjunta, pero la diferencia no es significativa $(\mathrm{V}=67$, $\mathrm{p}=.14 ; \mathrm{V}=55, \mathrm{p}=.54$ respectivamente). A los 42 meses se incrementan las unidades explicativas producidas por los niños $(\mathrm{V}=15$, $\mathrm{p}=.03$ ), quienes producen la mitad de las explicaciones.

El análisis del tipo de explicación (Figura 6) puso de manifiesto que a los 30

\section{Figura 4}

Iniciación de unidades explicativas según edad
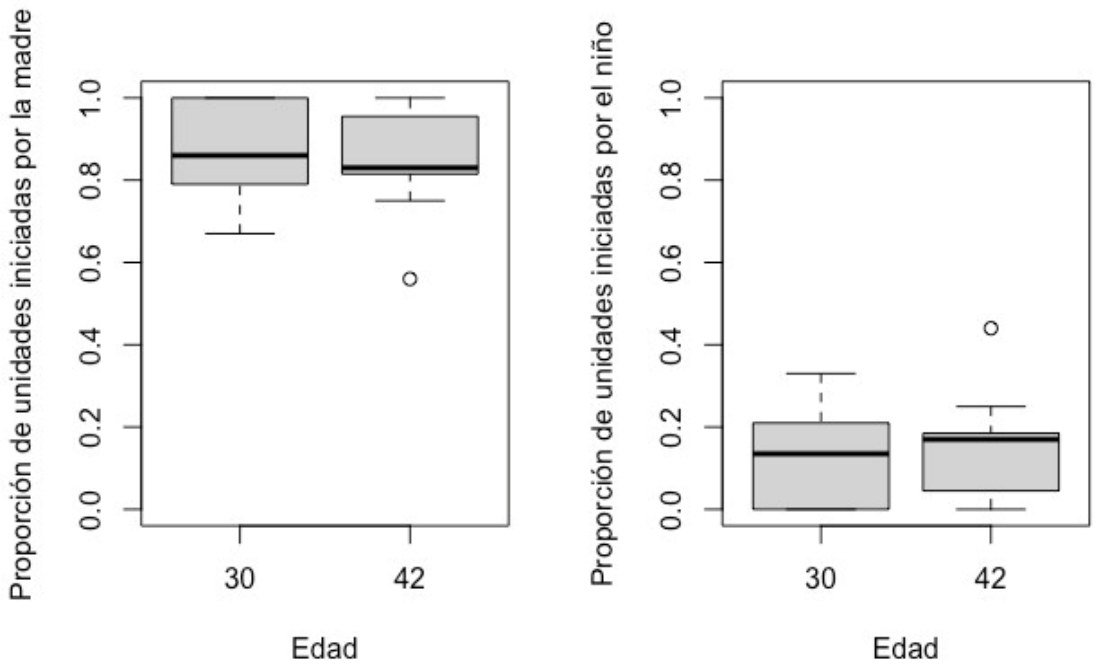

Revista de Psicología. Año 2021. Vol. 17, No 33, pp. 81-103 


\section{Figura 5}

Producción de las unidades explicativas según edad
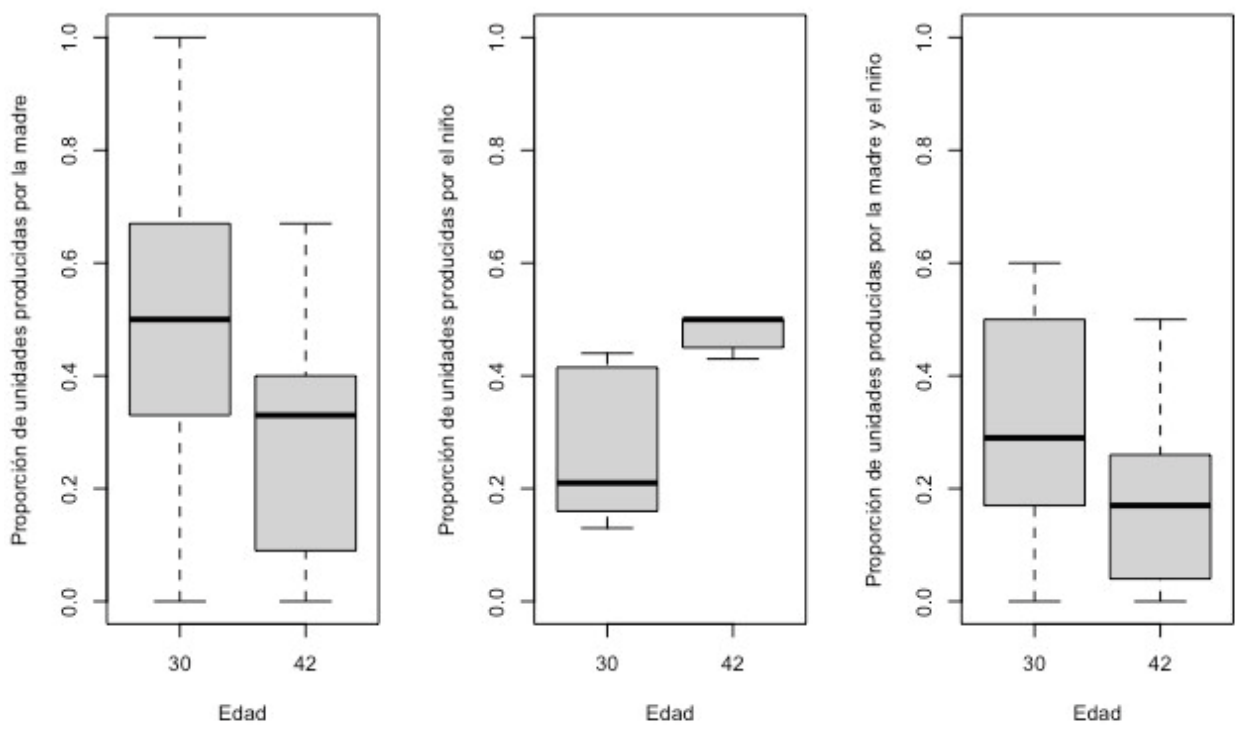

meses las explicaciones más frecuentes son las del tipo definición/descripción, seguidas de aquellas que expresan relaciones de causalidad. En conjunto, ambos tipos alcanzan casi el $90 \%$ de las unidades de discurso explicativas producidas en torno a la lectura. Cuando los niños tienen 42 meses, las explicaciones causales $(\mathrm{V}=83$, $\mathrm{p}=.006$ ), así como las que consisten en definiciones/ descripciones, descienden significativamente $\quad(\mathrm{V}=74, \quad \mathrm{p}=.04) . \quad \mathrm{En}$ este segundo momento se incrementan las unidades explicativas relacionadas con estados internos ( $\mathrm{V}=6, \mathrm{p}=.003)$. De manera conjunta, las explicaciones de tipo evidencial, procedimiento e intención se incrementan con la edad, pero alcanzan una proporción de 0,15 .

Por último, el análisis de las variaciones longitudinales en el habla explicativa durante la lectura de un cuento nuevo a los 42 meses también contempló el nivel de distanciamiento de las unidades de discurso explicativas (Figura 7).

A los 30 meses predominan las unidades explicativas de nivel bajo, seguidas de las de nivel alto y medio. A los 42 meses, las explicaciones de nivel alto son las más frecuentes, alcanzando una proporción de 0,51 . Se produce un leve descenso de las explicaciones de nivel medio. No se identificaron diferencias significativas en ninguno de los casos. 


\section{Figura 6}

Tipo de explicación según edad
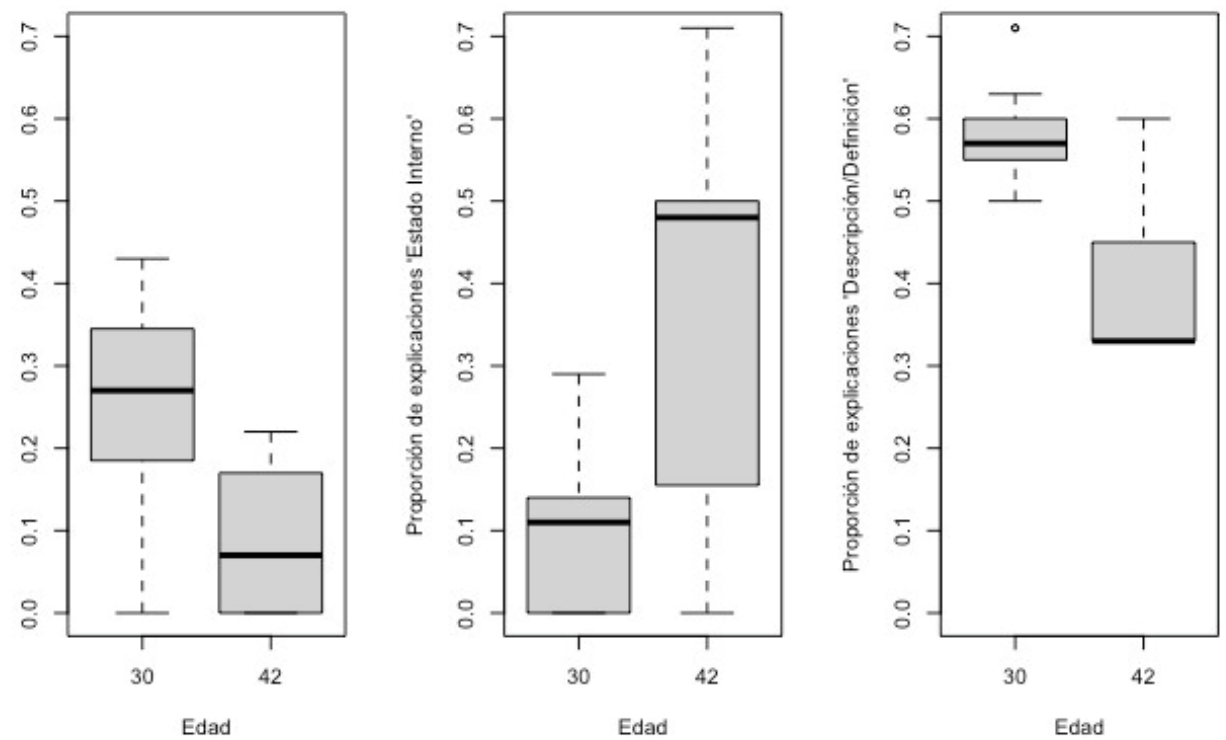

Figura 7

Nivel de distanciamiento de las unidades explicativas según edad

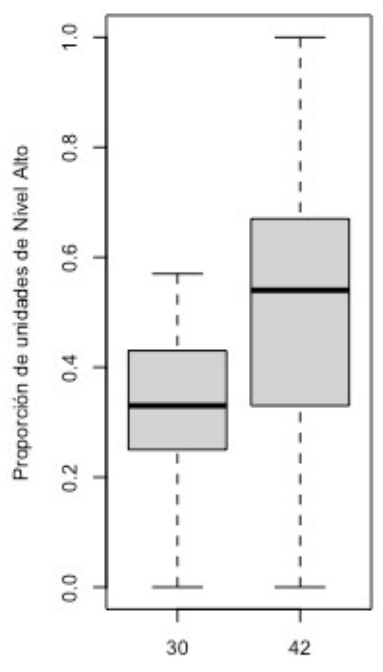

Edad
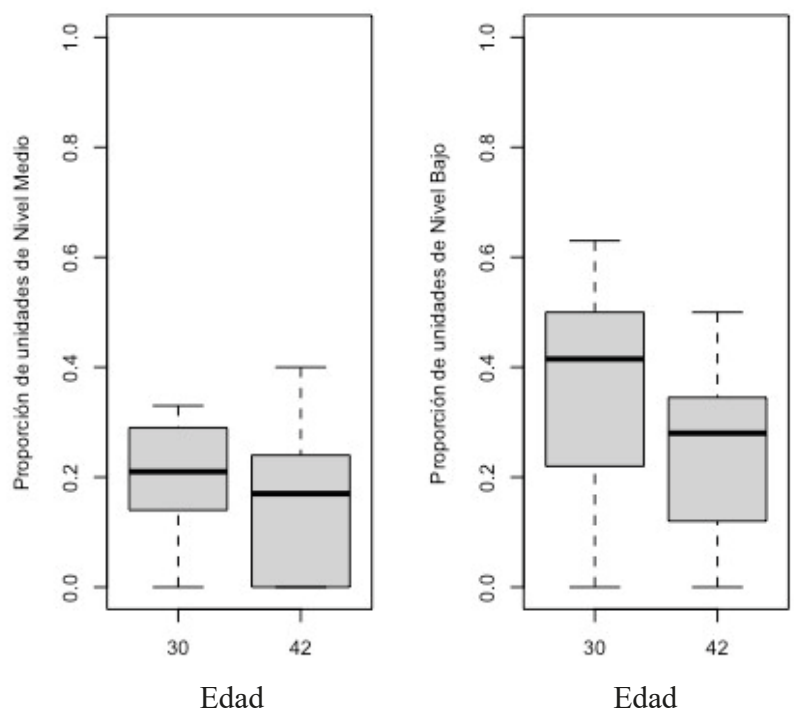

Edad

Revista de Psicología. Año 2021. Vol. 17, № 33, pp. 81-103 
¿Y esto qué es? ¿Por qué...? El desarrollo de unidades de discurso explicativas...

\section{Discusión y conclusiones}

El presente estudio se propuso contribuir al conocimiento del habla explicativa durante situaciones de lectura de cuentos en el hogar. De manera coincidente con hallazgos previos (Haden et al., 1996; Heath, 1983; Ninio \& Bruner, 1978; Stein \& Rosemberg, 2012; Sulzby \& Teale, 1987), se observó que las situaciones analizadas no consisten en una lectura literal del texto. Por el contrario, las madres y los niños producen verbalizaciones en torno al cuento que dan lugar a diferentes tipos de explicaciones. En efecto, el habla explicativa representa entre un $34 \%$ y un $39 \%$ del habla total producida durante las situaciones de lectura. Estas situaciones se caracterizan, asimismo, por su dialogicidad. Si bien las madres inician la mayoría de las unidades de discurso explicativas en las diferentes situaciones y momentos considerados, los niños - solos o con su madre - tienen un papel importante en su producción.

Estos resultados también coinciden con aquellos observados en investigaciones antecedentes focalizadas en el desarrollo del discurso explicativo, en tanto muestran que esta forma de discurso está presente en los intercambios cotidianos durante el período considerado (Aukrust, 2004; Aukrust \& Snow, 1998; Beals, 1993; Beals \& Snow, 1994; Blum-Kulka, 2002; Hickling \& Wellman, 2001; Veneziano \& Sinclair, 1985). Sin embargo, se observaron diferencias con los resultados de investigaciones previas que analizaron el habla explicativa durante otras actividades en el hogar, tanto en la misma población como en otros grupos. En este sentido, la proporción de habla explicativa durante la lectura es superior a la registrada en las situaciones de comida con población noruega, norteamericana, israelí (Aukrust, \& Snow, 1998; Blum-Kulka, 2002) y argentina (Stein et al., 2020). Asimismo, los hallazgos reportados en otras investigaciones pusieron de manifiesto que durante las comidas los niños se limitan mayormente a escuchar las explicaciones producidas por los adultos; durante las situaciones de lectura analizadas en el presente estudio, en cambio, muestran un papel activo en la producción de explicaciones, solos o con el apoyo adulto. Las lecturas de cuentos parecen facilitar, entonces, tanto la producción de habla explicativa como el papel del niño en su elaboración.

Las explicaciones más frecuentes en las situaciones de lectura analizadas expresan relaciones de causalidad, estados internos, y definiciones/descripciones de objetos, personajes y acciones. Estas explicaciones, promovidas por el objeto libro y la trama de la historia, están orientadas a comprender fenómenos más que a controlarlos, como se ha observado en las situaciones de comida analizadas en investigaciones previas (Beals, 1993).

Un aspecto importante del análisis realizado acerca de las unidades explicativas durante la lectura ha sido su nivel de distanciamiento, dada su relevancia para el desarrollo cognitivo (Sigel, 2002). De manera general, se observó que, en conjunto, predominan las explicaciones de nivel medio y alto, con diferencias longitudinales y entre situaciones que serán discutidas a continuación. Este hallazgo se diferencia de lo observado en otros estudios con respecto a las explicaciones generadas durante situaciones de juego (Aukrust,2004) y situaciones de comida (Stein et al., 2020), 
¿Y esto qué es? ¿Por qué...? El desarrollo de unidades de discurso explicativas...

donde predominaban las explicaciones ancladas en el "aquí y ahora". Las diferencias observadas pueden deberse al contexto de la actividad, a las relaciones de simetría / asimetría entre los participantes en cada caso, como así también a diferencias culturales entre los participantes (en el caso del estudio de Aukrust, 2004). Las características del habla explicativa en cuanto a su nivel de distanciamiento coinciden parcialmente con los resultados de Peralta y Salsa (2001). En su estudio con díadas madre-niño de distinto nivel socioeconómico de Argentina, observaron que las madres de sectores medios demandaban una participación activa a sus niños incentivándolos a elaborar sobre lo observable. Aunque este grupo de madres empleó más estrategias de nivel medio que sus pares de nivel socioeconómico bajo, privilegiaban al igual que ellas las estrategias de bajo distanciamiento. Es importante señalar que en el estudio mencionado no se consideraron las explicaciones de nivel alto, dado que los niños participantes no superaban los 24 meses de edad. Cabe pensar también que las diferencias se deben, en parte, al material de lectura empleado en cada estudio.

En cuanto a las variaciones longitudinales, se observó que, a los 42 meses, durante la lectura del mismo cuento que en el momento anterior, decrece la proporción de explicaciones iniciadas y producidas por la madre. Estas diferencias longitudinales no se mantienen durante la lectura de un cuento no conocido. La menor familiaridad con el cuento parece promover el protagonismo de la madre, incluso en las situaciones registradas cuando los niños tienen mayor edad. Por su parte, el papel del niño en la iniciación de las unidades de discurso explicativas se mantiene constante en los diferentes momentos y situaciones y no supera el 30\%. En cambio, se registró un incremento significativo de la participación del niño en la producción de explicaciones durante la lectura del cuento nuevo a los 42 meses. Dado que en estas situaciones las madres inician la mayor parte de las unidades explicativas, cabe pensar que los niños producen explicaciones en respuesta a preguntas formuladas por ellas; aspecto que se profundizará en futuros trabajos.

A diferencia de los resultados de investigaciones realizadas con población caucásica y afroamericana de habla inglesa en las que se observó un descenso en los intercambios durante la lectura con la edad (Heath, 1983; Heath \& Thomas, 1984; Sulzby $\&$ Teale, 1987), las situaciones analizadas en el presente estudio no parecen tornarse menos dialógicas. Por un lado, como se señaló, el habla explicativa en torno al cuento se mantiene constante. Por otro lado, las madres continúan iniciando unidades explicativas a lo largo del tiempo, y los niños, por su parte, incrementan su participación en la producción de explicaciones, especialmente durante la lectura del cuento nuevo a los 42 meses.

El análisis longitudinal mostró variaciones en relación con los tipos de explicaciones producidas en los intercambios. Durante la lectura del cuento a los 30 meses, predominan las explicaciones que consisten en definiciones y descripciones de los personajes, objetos y acciones presentes en el texto y en las ilustraciones. En los dos tipos de situaciones registradas a los 42 meses - lectura de un cuento conocido y lectura de un cuento nuevo- se observó un descenso significativo de este tipo de 
explicación. Ello coincide con estudios previos en los que se analizó la interacción en torno a la lectura en el hogar según la edad de los niños (Haden et al., 1996, Ninio \& Bruner, 1978; Van Kleeck et al., 1997). A pesar del descenso mencionado, este tipo de explicaciones resulta aún frecuente a los 42 meses, representando más del $30 \%$ de las unidades explicativas producidas tanto durante la lectura del mismo cuento como del cuento nuevo. La presencia de este tipo de explicaciones resulta relevante, dada su frecuencia en el contexto escolar. En efecto, se ha observado que la habilidad para proporcionar definiciones formales (Peets \& Bialystock, 2015) y descripciones claras (Ricard \& Snow, 1990) está asociada con el lenguaje académico y la escolarización.

Durante la lectura del cuento no conocido a los 42 meses se produce un descenso significativo en la proporción de explicaciones de causalidad, junto con un incremento también significativo de las unidades relativas a estados internos.

Estas variaciones pueden estar relacionadas con diferencias en la trama narrativa de los dos cuentos empleados en el estudio. Este aspecto constituye una limitación de la presente investigación, dado que no fue analizado de manera sistemática. Sin embargo, en el estudio de Peralta y Salsa (2001) con población argentina, se observó que el estilo de interacción de las madres de sectores medios no variaba significativamente según la complejidad del estímulo.

Con respecto al nivel de distanciamiento de las explicaciones, se registró un descenso de las unidades de nivel bajo a los 42 meses durante la lectura del cuento conocido. Si bien en la lectura del cuento nuevo se produce un incremento de las explicaciones de nivel alto, no resultó estadísticamente significativo. La mayor edad de los niños, junto con el conocimiento de la historia, parecen facilitar el uso de un habla más compleja y distanciada.

El presente estudio proporciona evidencia adicional acerca de las interrelaciones entre diferentes formas de discurso en las actividades cotidianas durante la infancia (Aukrust \& Snow, 1998; Beals \& Snow, 1994; Stein et al., 2020). De manera coincidente con estudios previos, se destaca el potencial de las situaciones de lectura de cuentos para promover la comprensión y producción de explicaciones diversas y complejas, de manera compartida entre el niño y el adulto (Heath, 1983; Kelemen et al., 2014; Leech et al, 2020; Legare et al., 2013). Ello tiene implicancias pedagógicas a considerar en el diseño de materiales e intervenciones educativas que contribuyan a incrementar las oportunidades para el dominio de diferentes formas de discurso extendido.

\section{Referencias}

Aukrust, V. (2004). Explanatory discourse in young second language learners' peer play. Discourse Studies, 6(3), 393-412. doi: 10.1177/14614456040

Aukrust, V. \& Snow, C. (1998). Narratives and explanations during mealtime conversations. Lang. in Soc., 27(2),
221-246.

Barbieri, M., Colavita, F. \& Scheuer, N. (1990). The beginning of the explaining capacity. En G. Conti Ramsden \& C. Snow (eds.), Children's language, (Vol.7, pp.245272). NJ: Erlbaum. 
Beals, D. (1993). Explanatory talk in low-income families' mealtime conversations. Applied Psycholinguistics, 14(4), 489-513. doi: 10.1017/ S0142716400010717

Beals, D. \& Snow, C. (1994) "Thunder is when the angels are upstairs bowling": Narratives and explanations at the dinner table. Journal of Narrative and Life History, 4, 331352.

Blum-Kulka, S.(2002). 'Do you believe that Lotís wife is blocking the Road 85 (to Jericho)?': Co-constructing theories about the world with adults En S. Blum-Kulka \& C. Snow (eds.), Talking to adults. The contribution of multiparty discourse to language acquisition (pp. 85-116). NJ: Erlbaum.

Blum-Kulka, S., Hamo, M. \& Habib, T. (2010) Explanations in naturally occurring peer talk: Conversational emergence and function, thematic scope, and contribution to the development of discursive skills. First Language, 30(3-4), 440-460.

Callanan, M. \& Oakes, L. (1992). Preschoolers' questions and parents' explanations: Causal thinking in everyday activity. Cognitive Development, 7(2), 213-233.

Crowley, K., Callanan, M., Tenenbaum, H. \& Allen, E. (2001). Parents explain more often to boys than to girls during shared scientific thinking. Psychol. Science, 12(3), 258-261.

Carmiol, A.M. \& Sparks, A. (2014). Narrative development across cultural contexts. En D. Matthews (ed.) Pragmatic development in first lan- guage acquisition (pp. 279-296). Benjamins.

Consejo Nacional de Investigaciones Científicas y Técnicas. (2006). Lineamientos para el comportamiento ético en las Ciencias Sociales y Humanidades, Resolución 2857/06. https://www.conicet.gov.ar/wp-content/uploads/RD-20061211-2857. pdf

Goodsitt, J.G., Raitan, M. \& Perlmutter, M. (1988). Interaction between mothers and preschool children when reading a novel and familiar book. International Journal of Behavioral Development, 11(4), 489-505. doi: 10.1177/016502548801100407

Griffin, T., Hemphill,L., Camp, L. \& Wolf, D. (2004). Oral discourse in the preschool years and later literacy skills. First Language, 24(2), 123-147. doi: $10.1177 / 0142723704042369$

Haden, C., Reese, E. \& Fivush, R. (1996). Mothers' extratextual comments during storybook reading: Stylistic differences over time and across texts. Discourse processes, 21(2), 135-169. doi: 10.1080/01638539609544953

Heath, S. (1983). Ways with words: Language, life, and work in communities and classrooms. Cambridge: Harvard Univ. Press.

Heath, S. \& Thomas,C. (1984). The achievement of preschool literacy for mother and child. En H. Goelman, A. Oberg \& F. Smith (eds.), Awakening to literacy (pp.51-72). Heinemann.

Heller, V. (2014). Discursive practices in family dinner talk and classroom discourse: A contextual comparison. 
¿Y esto qué es? ¿Por qué...? El desarrollo de unidades de discurso explicativas...

Learning, Culture and Social Interaction, 3(2), 134-145.

Hickling, A. \& Wellman, H. (2001). The emergence of children's causal explanations and theories: Evidence from everyday conversation. Dev. Psychology,3 7(5), 668-683.

Kelemen, D., Emmons, N., Seston Schillaci, R. \& Ganea, P. (2014). Young children can be taught basic natural selection using a picture-storybook intervention. Psychological Science, 25(4), 893-902. doi: 10.1177/0956797613516009

Leech, K., Haber, A., Jalkh, Y. \& Corriveau, K. (2020). Embedding scientific explanations into storybook impacts scientific discourse and learning. Frontiers in Psychology, 11:1016. doi: 10.3389/fpsyg.2020.01016

Legare, C. \& Clegg, J. (2014). The development of children's causal explanations. En S. Robson \& S. Flannery (eds.) The Routledge International Handbook of Young Children's Thinking and Understanding. Routledge Handbooks Online.

Legare, C., Lane, J. \& Evans, E. (2013). Anthropomorphizing science: How does it affect the development of evolutionary concepts? Merrill-Palmer Quarterly, 59(2),168-197.

McCabe, A., Bailey, A. \& Melzi, G. (2008). Spanish-language narration and literacy: Culture, cognition, and emotion. Cambridge: Cambridge Univ. Press.

MacWhinney, B. (2000). The CHILDES Project: Tools for Analyzing Talk. 3rd Edition. Mahwah: Erlbaum.

Nelson, K. (1989). Narratives from the crib.
Cambridge: Harvard Univ. Press.

Ninio, A. \& Bruner, J. (1978). The achievement and antecedents of labelling. Journal of Child Language, 5, 1-15.

Peralta O. \& Salsa A. (2001). Interacción materno-infantil con libros con imágenes en dos niveles socioeconómicos. Infancia y Aprendizaje, 24(3), 325-339.

Pan, B. \& Snow, C. (1999). The development of conversational and discourse skills. En M. Barrett, The development of language (pp.229-250). NY: Taylor \& Francis.

Pappas, C. (1993). Is narrative "primary"? Some insights from kindergartners' pretend reading of stories and informational books. Journal of Reading Behavior, 25, 97-127.

Peets, K. \& Bialystok, E. (2015). Academic discourse: Dissociating standardized and conversational measures of language proficiency in bilingual kindergarteners. Applied Psycholinguistics, 36(2), 437-461. doi:10.1017/S0142716413000301

Phillips, G. \& McNaughton, S. (1990). The practice of storybook reading to preschool children in mainstream New Zealand families. Reading Research Quarterly, 25(3), 196-212.

Quasthoff, U., Heller, V. \& Morek, M. (2017). On the sequential organization and genre-orientation of discourse units in interaction: An analytic framework. Discourse Studies, 19(1), 84-110. doi: $10.1177 / 1461445616683596$

R Core Team (2017). R: A language and environment for statistical computing. https://www.r-project.org/ 
Ricard, R. \& Snow, C. (1990). Language use in and out of context. Journal of Applied Dev. Psychology, 11(3), 251-266.

Sepúlveda, A. \& Álvarez-Otero, B. (2010) El aprendizaje de los usos expositivos del lenguaje. Cs. de la Salud, 8(2), 45-59.

Sigel, I. E. (2002). The psychological distancing model: A study of the socialization of cognition. Culture and Psychology, 8(2), 189-214. doi: 10.1177/1354067X02008002438

Snow, C. (2014). Input to interaction to instruction: Three key shifts in the history of child language research. Journal of Child Language, 41, 117-123.

Snow, C. \& Beals, D. (2006). Mealtime talk that supports literacy development. New directions for child and adolescent development, 111, 51-66.

Snow, C. \& Uccelli, P. (2009). The challenge of academic language. En D. Olson $\&$ N. Torrance (eds.), The Cambridge handbook of literacy (pp.112133). Cambridge Univ. Press.

Stein, A., Migdalek, M. \& Rosemberg, C. R. (2020) Argumentación, narración y explicación: un estudio de las unidades de discurso en conversaciones espontáneas durante situaciones de comida. Cultura y Educación, 32(4), 705-737.

Stein, A. \& Rosemberg, C. R. (2012). Compartir cuentos en el ho- gar. Diferentes estilos de lectura en poblaciones urbano marginadas de Argentina. Infancia $y$ aprendizaje, 35(4), 405-419. doi:10.1174/021037012803495249

Sulzby, E. \& Teale, W. (1987). Young children's storybook reading: Longitudinal study of parent-child interaction and children's independent functioning. Spencer's Found. Report.

Van Kleeck, A., Gillam, R.,Hamilton, L. \& McGrath, C.(1997). The relationship between middle-class parents book-sharing discussion and their preschoolers abstract language development. Journal of Speech, Lang. and Hearing, 40, 1261-1271.

Veneziano, E. \& Sinclair, H. (1995). Functional changes in early child language: the appearance of references to the past and of explanations. Journal of Child Lang.,22, 557-581.

Wells, G. (1985). Preschool literacy-related activities and success in school. En D. Olson, N. Torrance \& A. Hildyard (eds.) Literacy, language and learning (pp. 229-255). NY: Cambridge Univ. Press.

Zunino, G. M. (2017). Procesamiento psicolingüístico de relaciones causales y contracausales. En I. Arroyo Hernández (ed.). La expresión de la causa en español (pp.199-234). Madrid: Visor. 


\section{Notas al final}

${ }^{1}$ Los datos analizados en el presente estudio forman parte de un corpus más amplio recogido en el marco del proyecto "Narración y alfabetización temprana. Un estudio longitudinal de situaciones de interacción de las que participan niños de distintos grupos sociales en su medio familiar" aprobado por CONICET y con apoyo del subsidio PICT 20140553. Para el relevamiento del corpus mayor se realizó una visita cada seis meses al hogar de cada niño entre los 2.6 y los 5 años de edad. En cada visita se indujeron situaciones de juego, cuento y conversaciones realizadas conjuntamente por el niño y un miembro del hogar a cargo de su cuidado.

${ }^{2}$ Concebimos a la causalidad como una relación diádica entre dos entidades -propiedades, objetos, eventos, objeto/evento-: causa y efecto (Zunino, 2017). De esta manera, aunamos las explicaciones causales y consecutivas en una sola categoría: causalidad. 